\title{
Perinatal Outcomes in a Cohort of Offspring Exposed to Methadone Maintenance Therapy (MMT) in Utero
}

\author{
Christy Pylypjuk, BSC, MD, MSc, FRCSC $C^{1,2,3^{*}}$ and Romina Levy, BSc, MD \\ ${ }^{1}$ Department of Obstetrics, Gynecology and Reproductive Sciences, University of Manitoba, Canada \\ ${ }^{2}$ Children's Hospital Research Institute of Manitoba, Canada \\ ${ }^{3}$ HSC Women's Hospital, Canada \\ ${ }^{4}$ Department of Obstetrics and Gynecology, University of British Columbia, Canada
}

\begin{abstract}
Objective: To determine the perinatal outcomes of offspring exposed to methadone maintenance treatment (MMT) in utero.

Methods: This was a historical, matched-cohort study at a tertiary obstetrical hospital (2010-2015). Controls were matched to 'cases' of newborns exposed to MMT in utero in a 2:1 fashion according to maternal age, neighborhood, and year of delivery. Maternal and newborn hospital charts were reviewed in order to collect relevant demographic information as well as the perinatal outcomes of interest. Descriptive statistics and comparative analyses were performed to determine differences in outcomes between groups.

Results: There were 363 newborns in the cohort (121 MMT cases and 242 controls). There was no difference in basic demographics between groups, however mothers in the MMT group were more likely to smoke cigarettes and receive treatment for mental illness. Newborns in the MMT group had an almost 3-fold higher incidence in preterm birth than controls. Even after stratifying by gestational age and maternal smoking, newborns in the MMT group had consistently lower birthweights, higher NICU admissions, and longer in-patient stays when compared to matched controls. However, there was no difference in peripartum complications, Apgar scores, growth restriction, or overall survival.

Conclusion: When compared to matched-controls, MMT exposure in utero is associated with earlier delivery, lower birthweight, and higher neonatal intermediate- and intensive-care unit admissions. However, there is no difference in overall survival to discharge, intrapartum complications, or major morbidity. Larger studies with the capacity to adjust for potential sociodemographic covariates are still needed to better elucidate the relationship between MMT exposure in utero and neonatal outcomes beyond neonatal withdrawal. The high rates of prolonged in-patient, NICU stays offer an opportunity for exploring alternate models of postnatal in-patient care in order to improve outcomes and reduce the burden associated with neonatal withdrawal after MMT.
\end{abstract}

Keywords

Methadone, Opioid dependence, Substance abuse in pregnancy

\section{Introduction}

There has been a steady increase in substance abuse and opioid addiction over the past decade [1]. This trend has also been seen amongst pregnant women, and now with heightened concerns about the intergenerational effects of addictions including future health of offspring $[2,3]$. For pregnant women in the U.S, the rate of admission to drug treatment services for prescription opioid abuse increased drastically from $2 \%$ in 1992 to $28 \%$ in 2012 [2]. These numbers are consistent with other population data showing that opioid abuse is currently the most common reason for seeking addictions treatment for illicit substance use during pregnancy, followed by marijuana and other substances $[4,5]$. The incidence of infants born to mothers using opioids or having an opioid dependence increased from 1.19 to 5.63 per 1000 hospital live births per year from the year 2000 to 2009 [6]. For women with an opioid dependence in pregnancy, management

*Corresponding author: Christy Pylypjuk, BSc, MD, MSc, FRCSC, HSC Women's Hospital, 820 Sherbrook Street, Winnipeg, R3A 1R9, Canada

Accepted: February 24, 2020

Published online: February 26, 2020

Citation: Pylypjuk C, Levy R (2020) Perinatal Outcomes in a Cohort of Offspring Exposed to Methadone Maintenance Therapy (MMT) in Utero. Clin Pediatr Res 4(1):63-71 
options include methadone maintenance treatment (MMT) or complete opioid detoxification [4-8]. The mechanism of methadone, a synthetic opiate, is as a competitive agonist at the mu-opioid receptor $[8,9]$. It also functions as a competitive inhibitor of the glutamate receptor within the central nervous system to lessen the symptoms of opioid withdrawal. Important benefits of methadone for opioid addiction management include reducing the risk of death, infectious diseases, as well as improving mental health and social/relational stability $[5,9,10]$. Because of the established benefit and safety of methadone during pregnancy, it is recommended that pregnant women with opioid dependence be immediately referred to an addiction specialist in order to facilitate prompt access to MMT $[7,9,10]$. Maternal involvement in a stable, medically monitored MMT program also improves attendance for prenatal care, which also leads to better pregnancy outcomes [11-17].

Recent studies have demonstrated that complete detoxification in opioid-dependent pregnant women is not associated with harmful outcomes for the fetus or obstetrical complications, especially when done in the second and third trimesters $[11,12,14-17]$. But because opioid detoxification during pregnancy is associated with high relapse rates, which is itself harmful to the developing fetus, current national practice guidelines advocate for use of MMT for management of opioid dependence in pregnancy [7]. As a harm-reduction strategy, adherence to MMT in pregnancy is particularly impactful at reducing relapse to illicit opioid use $[7,12,13,17]$.

With higher rates of opioid dependence in pregnancy, it is not surprising that there has been a simultaneous increase in rates of neonatal opioid withdrawal syndrome (NOWS) over the same time period [18]. NOWS, formerly known as 'neonatal abstinence syndrome' or 'NAS', refers to the postnatal withdrawal seen in some newborns following exposure to maternal opioid use in utero $[11,12,18,19]$. NOWS is characterized by excessive high-pitched cry/irritability, sleep-wake disturbances, alterations in infant tone and movement, feeding difficulties, gastrointestinal disturbances, autonomic dysfunction, and failure to thrive [11-14]. Treatment of NOWS has historically centered around postnatal administration of morphine and other opioid agonists to affected newborns $[11,12,14]$. Such treatment also frequently requires lengthy in-patient hospital stays and intensive neonatal care. However, there is emerging evidence that providing enhanced postpartum care to mothers including supports for breastfeeding, skin-to-skin care, swaddling, and rooming-in can all help improve maternal-newborn bonding and reduce the sequelae associated with withdrawal symptoms in the newborn [20-26]. Other neonatal outcomes associated with maternal opioid use, including MMT, include increased rates of prematurity, small-for-gestational age neonates [15], and admission to neonatal intensive care units (NICUs) [16]. However, because of conflicting evidence in the literature about the exact magnitude of risk, if any, for offspring exposed to MMT as well as general misinformation in the public, many women are advised or independently choose to quit MMT during pregnancy.
While the pharmacologic safety profile of MMT in pregnancy has been established, much of the literature about neonatal outcomes exists in relation to NOWS and related sequelae, and there remains a paucity of information about pregnancy and other neonatal/pediatric outcomes following MMT exposure in utero [2]. There is also inconsistency in the literature regarding the relationship, if any, between dose of MMT and outcomes. To date, systematic reviews and meta-analyses attempting to clarify these associations have been limited by heterogeneity of small sample sizes of primary studies $[2,27]$. Observational studies attempting to evaluate the impact of methadone exposure in utero by comparing cases of maternal MMT to the general obstetrical population are fraught with sociodemographic confounders inherent to studying a population with multiple risk factors for comorbidities and adverse health outcomes. As such, there remains the need for ongoing research into the impact of MMT for mothers and offspring alike.

The purpose of our study was to describe the perinatal outcomes, aside from NOWS, of offspring exposed to MMT in utero, as compared to matched controls that were not exposed to MMT. By accounting for potential socioeconomic confounders, this study aimed to isolate the effects of MMT on newborns beyond NOWS in an effort to improve targeted neonatal and pediatric care.

\section{Methods}

This was a historical, matched-cohort study of singleton births at a tertiary obstetrical hospital in Canada. Over the 5-year study period (2010 to 2015), all live born singletons delivering at the hospital were eligible for inclusion. 'Cases' of in utero MMT exposure were identified using an in-patient pharmacy database of maternal prescriptions, and then used to link the maternal record to the offspring chart. Using concurrent sampling methods, a control group was assembled from those newborns not exposed to MMT in utero and individually-matched to 'cases' in a 2:1 fashion according to maternal age, neighborhood, and year of delivery. Neighborhood was defined using forward sortation area from mailing address postal codes and was used as a surrogate for socioeconomic status. Maternal age was also chosen as a matching variable given its known association with adverse perinatal outcomes [28]. Newborns were excluded if postnatal palliation was planned, stillbirth prior to labor, or if their primary residence was outside the hospital catchment area (and thus precluded our ability to identify matched controls). This study was also restricted to singleton pregnancies given that twins and higher-order multiples have an inherently higher risk of perinatal complications and our inability to account for chorionicity during the matching process might have compromised the comparability of groups [29].

Using a standardized data collection sheet, the maternal and newborn hospital records were reviewed in order to collect information about maternal demographics, obstetrical events, and neonatal outcomes. The incidence of MMT exposure in pregnancy was calculated using the total number of live births at the hospital over the study 
period. Maternal body mass index (BMI) was collected as a continuous variable and by using standardized BMI categories (underweight, normal, overweight, obese) [30]. Methadone dose was recorded as a continuous variable in milligrams. Given inconsistencies in the literature regarding the definition of low-, moderate- and high-dose methadone, a decision was made a priori to use either the mean or median methadone dose of women in the MMT group, depending on the distribution, to define the cutoff between low and high-dose methadone in this study.

Birthweight was described as continuous variable as well as categorically according to standardized growth curves used in local practice and by the following gestational age-specific cutoffs: 'Small for gestational age' was defined as birthweight below the $10^{\text {th }}$ percentile for gestational age; 'large for gestational age' was defined as birthweight above the $90^{\text {th }}$ percentile for gestational age [31]. Continuous variables were described as means with standard deviations if normally distributed or as medians with interquartile ranges if non-parametrically distributed.
Dichotomous and categorical variables were described as proportions. The Chi-square test was used to compare dichotomous variables between groups (MMT vs. nonMMT). Means were compared using either the student t-test (if normally distributed) or the Wilcoxon rank sum test (if non-normal distribution). Stratification and regression analysis were used to account for potential confounders. A $p$-value of $<0.05$ was used to define statistical significance. Statistical analysis was performed using Stata v.14.2 (Stata Corp LLC, College Station, TX) software.

\section{Results}

Over the 5-year study period, there were 27,601 live births at our center. Initially, 192 cases of MMT were identified from in-patient pharmacy records by maternal methadone prescriptions. Of these, 71 cases were excluded based on the following criteria: Non-obstetrical patients (33); residence outside the hospital catchment area (32); and twins (3). The remaining 121 cases of MMT were included in the study and used to identify 242 matched controls as per the methods described above, in order to link maternal and new-

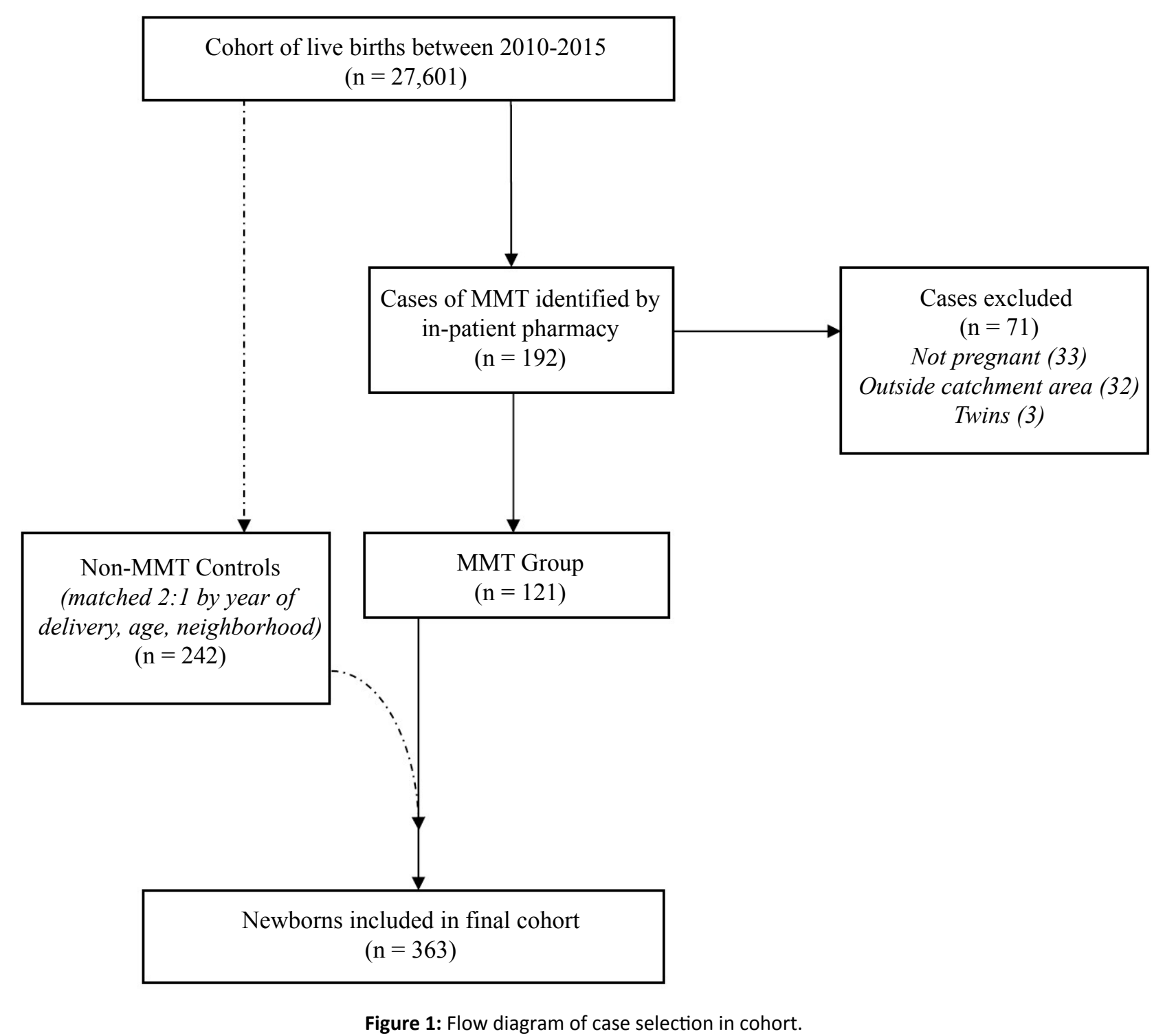


Citation: Pylypjuk C, Levy R (2020) Perinatal Outcomes in a Cohort of Offspring Exposed to Methadone Maintenance Therapy (MMT) in Utero. Clin Pediatr Res 4(1):63-71

Table 1: Maternal characteristics between groups, described as means (+/- standard deviation), medians [interquartile range], or proportions (\%).

\begin{tabular}{|c|c|c|c|}
\hline & MMT Group $(n=121)$ & Controls $(n=242)$ & $p$-value \\
\hline Maternal age, mean (SD) & $28.6(5.0)$ & $28.6(5.0)$ & 0.96 \\
\hline Body Mass Index, mean (SD) & $31.3(4.9)$ & $31.4(6.5)$ & 0.88 \\
\hline \multicolumn{4}{|l|}{ Parity } \\
\hline Multiparous, $\%$ & 83.5 & 75 & 0.17 \\
\hline Primiparous, $\%$ & 16.5 & 25 & 0.23 \\
\hline \multicolumn{4}{|l|}{ Residence (by postal code) } \\
\hline Inner City, \% & 51.2 & 51.2 & 1 \\
\hline Urban, \% & 41.3 & 41.3 & 1 \\
\hline Rural, \% & 7.4 & 7.4 & 1 \\
\hline Cigarette smoking, $\%$ & 89.8 & 32.8 & $<0.001$ \\
\hline Alcohol use, \% & 10.7 & 2.5 & 0.002 \\
\hline Mental Illness, \% & 36.4 & 25.6 & 0.04 \\
\hline Methadone Dose (in mg), median [IQR] & $70[44-100]$ & -- & -- \\
\hline
\end{tabular}

Table 2: Newborn outcomes between groups, described as means (+/- standard deviation), medians [interquartile range], or proportions (\%).

\begin{tabular}{|c|c|c|c|}
\hline & MMT Group $(n=121)$ & Controls $(n=242)$ & $p$-value \\
\hline GA at delivery, median [IQR] & $38+6[37-40]$ & $39+4[38+1-40+3]$ & 0.003 \\
\hline Preterm Birth, $\%$ & 19.8 & 7.4 & 0.001 \\
\hline \multicolumn{4}{|l|}{ Mode of delivery } \\
\hline Spontaneous VD, \% & 73.6 & 74.4 & 0.89 \\
\hline Operative VD, \% & 9.1 & 7.4 & 0.68 \\
\hline Cesarean Section (CS), \% & 17.3 & 18.2 & 0.83 \\
\hline Birthweight (in grams), mean (SD) & $3110.6(558.6)$ & 3374.0 (520.9) & $<0.001$ \\
\hline Small for gestational age, $\%$ & 5.1 & 3.4 & 0.44 \\
\hline 1-minute Apgar, median [IQR] & $8[8,9]$ & $8[8,9]$ & 0.64 \\
\hline 5-minute Apgar, median [IQR] & $9[9,9]$ & $9[9,9]$ & 0.89 \\
\hline \multicolumn{4}{|l|}{ Peripartum Complications } \\
\hline Fetal Distress in Labour, \% & 10.7 & 9.5 & 0.71 \\
\hline Emergency Delivery by CS, \% & 13.2 & 10.7 & 0.49 \\
\hline Neonatal Resuscitation, \% & 11.2 & 7.8 & 0.35 \\
\hline Admission to Neonatal Care Unit, \% & 90.9 & 7 & $<0.001$ \\
\hline Length of Hospital Stay, median [IQR] & $2[2,5]$ & $2[1,3]$ & 0.04 \\
\hline Prolonged Hospital Stay > $48 \mathrm{hrs}, \%$ & 40.5 & 26 & 0.01 \\
\hline
\end{tabular}

born records (Figure 1). The incidence of MMT in pregnancy at our hospital was $0.6 \%$ over the study period.

Regarding the overall characteristics of the pregnant women in this cohort, the mean maternal age was 28.6 years (SD 5.0). Most women were multiparas (79.3\%), compared to $20.7 \%$ who were primiparas. The median gestational age at delivery was 39 weeks +2 days [IQR 38 to $39+6$ ]. The mean $\mathrm{BMI}$ was $31.4 \mathrm{~kg} / \mathrm{cm}^{2}$. Using standardized BMI categories, there were no mothers that were underweight and only $8.6 \%$ of mothers had a normal BMI; over $90 \%$ of mothers in the cohort were either overweight or obese $(40.6 \%$ and $50.8 \%$ respectively). A high proportion of mothers were cigarette smokers (48.1\%) and $4.9 \%$ reported alcohol use during pregnancy. 33.2\% of mothers reported issues with mental health including depression, anxiety, and/or mood disorders other than addictions. Most women (92.5\%) were living in an urban area, and over half of these resided in an inner-city neighborhood (51.2\%).

While there were no significant differences in maternal age, residence, or parity between groups, mothers in the MMT group had significantly higher rates of smoking ( $89.8 \%$ vs. $32.5 \%, p<0.001)$, alcohol use $(10.7 \%$ vs. $2.5 \%, p$ $=0.002$ ), and preexisting mental illness (other than addictions) $(36.4 \%$ vs. $25.6 \%, p=0.04)$ when compared to con- 
Citation: Pylypjuk C, Levy R (2020) Perinatal Outcomes in a Cohort of Offspring Exposed to Methadone Maintenance Therapy (MMT) in Utero. Clin Pediatr Res 4(1):63-71

trols (Table 1). Amongst mothers in the MMT group, the median methadone dose was $70 \mathrm{mg}$ [IQR 44 to 100]. The incidence of intrapartum fetal distress $(10.7 \%$ versus $7.9 \% ; p$ $=0.38)$ and emergency delivery by cesarean section $(13.2 \%$ versus $10.7 \% ; p=0.48$ ) were similar in both groups (Table 2). For newborns, the gestational age at delivery was significantly different between groups; 38 weeks +6 days [IQR 37 to 40] in the MMT group versus 39 weeks +4 days [IQR $38+1$ to $40+3]$ in the control group $(p<0.003)$. There was almost a 3 times higher incidence of preterm birth prior to 37 weeks gestation in the MMT group compared to controls $(19.8 \%$ vs. $7.4 \%, p<0.001)$. Birthweight was also significantly different between groups, as newborns in the
MMT group had a mean weight of 3110.6 grams (SD 558.2) compared to 3374 grams (SD 520.9) $(p<0.001)$. The incidence of SGA was similar between groups (5.1\% vs. $3.4 \% ; p$ $=0.44$ ) (Table 2). However, more newborns in the control group were LGA at birth $(49.8 \%$ vs. $28 \% ; p<0.0001)$ (Figure 2 ). After stratification by maternal risk factors (smoking, methadone dosage, age), these differences in birthweights between groups were preserved with newborns exposed to MMT in utero having consistently lower birthweights than unexposed controls (Table 3). This trend was also seen amongst term and preterm newborns alike.

There was no difference in mode of delivery between

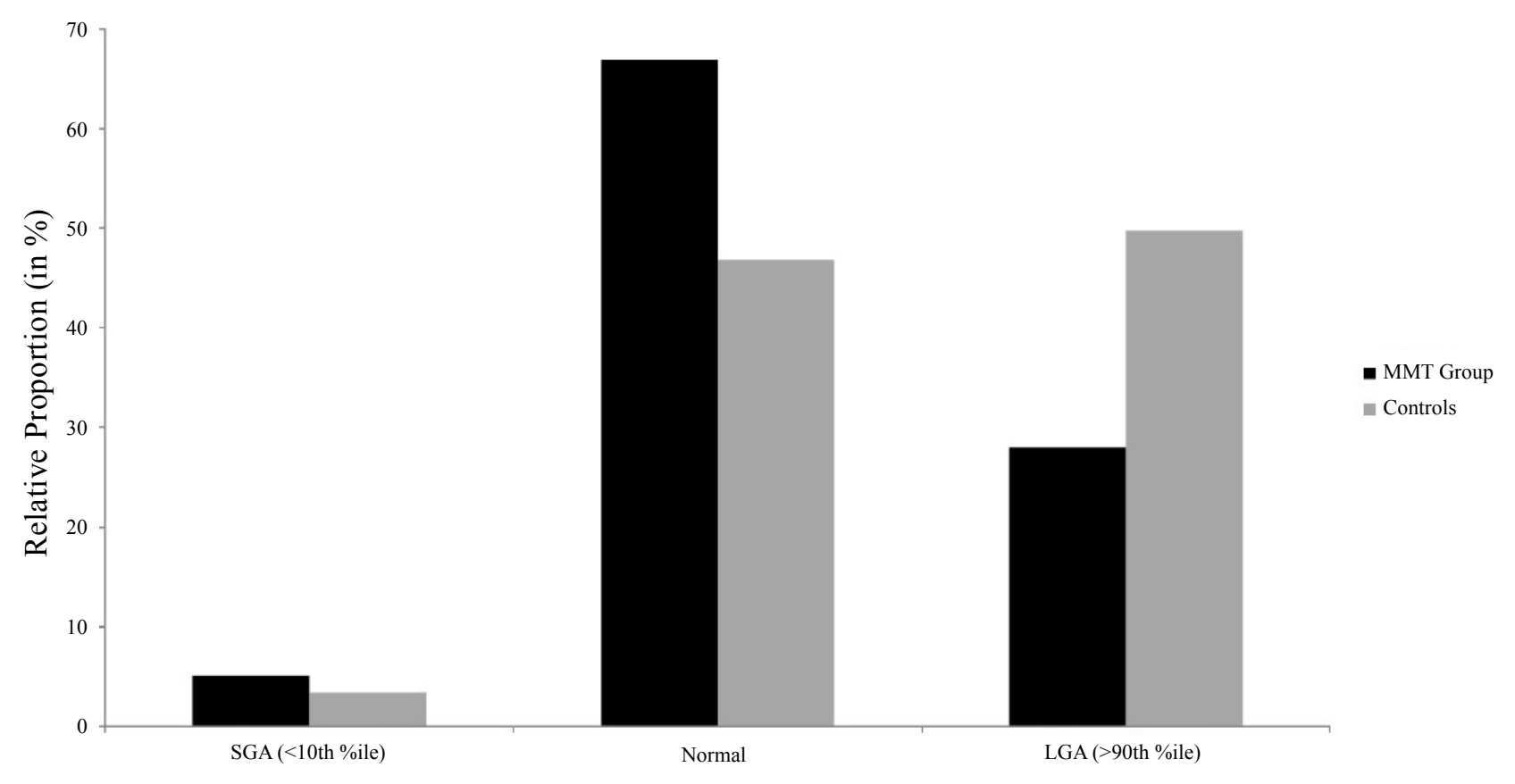

Figure 2: Birthweight categorized as small for gestational age (SGA) less than the $10^{\text {th }}$ percentile, normal, and large for gestational age (LGA) greater than the $90^{\text {th }}$ percentile.

Table 3: Neonatal birthweight (in grams) between groups, after stratification by maternal risk factors, and reported as a mean (standard deviation) or median [interquartile range].

\begin{tabular}{|c|c|c|c|}
\hline & MMT Group & Controls & $p$-value \\
\hline \multicolumn{4}{|l|}{ By Gestational Age Category } \\
\hline Term Deliveries & $3221.6(487.4)$ & $3432.2(474.7)$ & $<0.001$ \\
\hline Preterm Deliveries & $2239[2157,2825]$ & $2565[2310,3070]$ & 0.03 \\
\hline \multicolumn{4}{|l|}{ By Smoking Status } \\
\hline Non-smokers & $3104.9(575.0)$ & $3446.6(488.3)$ & $<0.001$ \\
\hline Smokers & $3052.0(568.5)$ & 3397.4 (442.9) & $<0.001$ \\
\hline \multicolumn{4}{|l|}{ By Methadone Dose (High vs Low) } \\
\hline High-Dose Methadone ( $\geq 70 \mathrm{mg}$ ) & 3023.8 (571.9) & -- & \\
\hline Low-Dose Methadone (< 70 mg) & $3109.5(558.0)$ & -- & $0.07^{*}$ \\
\hline \multicolumn{4}{|l|}{ By Maternal Age } \\
\hline Advanced Maternal Age ( $\geq 35$ ) & $3067.2(566.7)$ & $3360.0(496.7)$ & 0.03 \\
\hline Young Maternal Age $(<18)^{\wedge}$ & -- & -- & -- \\
\hline
\end{tabular}


Citation: Pylypjuk C, Levy R (2020) Perinatal Outcomes in a Cohort of Offspring Exposed to Methadone Maintenance Therapy (MMT) in Utero. Clin Pediatr Res 4(1):63-71

groups, as most births occurred via spontaneous vaginal delivery (73.7\% vs. $74.3 \% ; p=0.89$ ) (Table 2 ). There was no difference in need for emergency delivery by Cesarean section for fetal distress ( $13.2 \%$ vs. $10.7 \% ; p=0.49)$ nor in the need for immediate postnatal resuscitation between groups (11.2\% vs. $7.8 \% ; p=0.35)$. Apgar scores at 1 -minute ( 8 versus $9 ; p=0.64$ ) and 5 -minutes (9 versus $9 ; p=0.89$ ) were also similar between groups. However, newborns in the MMT group had a neonatal intermediate- or intensive-care unit admission rate over $90 \%$, compared to only $7 \%$ for controls $(p<0.001)$ owing to complications of neonatal opioid withdrawal and local practice policies regarding surveillance for NOWS. Overall, the median length of stay in hospital for newborns in the cohort was 2 days [IQR 1 to 3], although there was a significant difference in median stay between groups $(p=0.04)$. In the MMT group, the median length of stay for newborns was 2 days [IQR 2 to 5] with a range of stays from 1 to 62 days. Of note, $40.5 \%$ of newborns in the MMT group had a prolonged hospital stay of $>48$ hours, the bulk of which was spent in observation in a neonatal intermediate- or intensive-care unit. However, it meant that $59.5 \%$ of newborns in the MMT group required less than 48 hours in hospital. For newborn controls, the median length of stay was 2 days [IQR 1 to 3] with a range in stays from 1 to 6 days. 26\% of controls required admission to hospital for $>48 \mathrm{hrs}$ while the majority of controls $(74 \%)$ were discharged from hospital in under 2 days. There were no neonatal deaths in either group and all newborns survived to discharge from hospital. It should be noted that there was no evidence of a relationship between maternal methadone dosage (high-dose $\geq 70 \mathrm{mg}$ vs. low-dose $<70 \mathrm{mg}$ ) and neonatal outcomes including gestational age, preterm birth, peripartum complications, or admission to neonatal intermediate- or intensive-care units (Table 4). There was also no evidence of a difference in median length of hospital stay by maternal methadone dosage, although there was trend toward more prolonged hospital stays amongst offspring born to mothers on lowdose methadone (48.8\% vs. $35.2 \%$; $198 p=0.15$ ).

\section{Discussion}

This study provides valuable insights into perinatal outcomes, beyond NOWS, for newborns exposed to MMT in utero as compared to matched controls. The baseline demographic characteristics of our study groups were not significantly different, validating our successful matching of cases to controls. There were also no differences in parity between the two groups, further demonstrating equivalency between groups and strengthening the reliability of our results. Use of neighborhood matches by postal code attempted to account for additional sociodemographic variables which may influence perinatal outcomes in this population. Prior studies which have compared outcomes between newborns with MMT exposure in utero have typically drawn controls from the general obstetrical population. However, given the predisposition of women on MMT in pregnancy to have other social disparities compared to other pregnant women, it can be difficult to isolate in utero methadone exposure as the sole determinant of adverse perinatal outcomes.

When comparing rates of cigarette smoking between mothers on MMT and controls, mothers in the MMT group had significantly higher rates of smoking compared to controls. This is consistent to previous literature that reports high smoking rates among participants of MMT [32-35]. The detrimental effects of cigarette smoking during pregnancy have been reported extensively including various antenatal obstetrical complications as well as worsened fetal and neonatal outcomes (fetal growth restriction and low birth weight, increased perinatal mortality, childhood asthma, and long-term behavioral problems) [36,37]. However, there was no evidence that smoking confounded our results; newborns exposed to smoking had lower birthweights in both groups, and even lower if there was concurrent exposure to MMT. Smoking was also not significantly correlated with preterm birth in this sample.

While the effects of methadone appear separate from

Table 4: Neonatal outcomes between groups, after stratification by methadone dose.

\begin{tabular}{|c|c|c|c|}
\hline & High-Dose $\geq 70 \mathrm{mg}$ & Low-Dose $<70 \mathrm{mg}$ & $p$-value \\
\hline \multicolumn{4}{|l|}{ Gestational Age } \\
\hline Gestational Age, mean (SD) & $267.3(16.7)$ & $271.3(18.6)$ & 0.24 \\
\hline Preterm Birth, $\%$ & 19.5 & 20 & 0.95 \\
\hline \multicolumn{4}{|l|}{ Peripartum Complications } \\
\hline Nonreassuring fetal status in labour, \% & 10.2 & 12.2 & 0.74 \\
\hline Emergency Cesarean Delivery, \% & 12.5 & 12.2 & 0.96 \\
\hline 1-minute Apgar, median [IQR] & $8[8,9]$ & $8[8,9]$ & 0.78 \\
\hline 5-minute Apgar, median [IQR] & $9[9,9]$ & $9[9,9]$ & 0.91 \\
\hline Neonatal Resuscitation, $\%$ & 10.2 & 12.2 & 0.74 \\
\hline Admission to Neonatal Care Unit, $\%$ & 95.5 & 87.8 & 0.12 \\
\hline \multicolumn{4}{|l|}{ Length of Hospital Stay } \\
\hline Length of Stay, median [IQR] & $2[2,4]$ & $2[2,5]$ & 0.35 \\
\hline Prolonged Hospital Stay, \% & 35.2 & 48.8 & 0.15 \\
\hline
\end{tabular}


Citation: Pylypjuk C, Levy R (2020) Perinatal Outcomes in a Cohort of Offspring Exposed to Methadone Maintenance Therapy (MMT) in Utero. Clin Pediatr Res 4(1):63-71

the adverse outcomes of smoking, smoking is still a very serious issue during pregnancy with implications for offspring exposed in utero plus postnatally. Considering this, and previous evidence demonstrating high rates of readiness to quit smoking in MMT participants, pregnancy still offers a critical window of opportunity for smoking cessation interventions for mothers on MMT.

When we looked at preterm birth we found statistical significance similar to other studies, with methadone patients delivering prior to 37 weeks gestation at a rate of almost three times that of the control group. Mothers on MMT should be counseled about this risk accordingly, as well as associated sequelae of prematurity. Of note, most of the preterm births in our sample occurred during the late preterm period as opposed to early preterm or at borderline viability. While the impact of late prematurity is not insignificant, survival is high and long-term morbidity is low. Risk of late prematurity should not be a reason to withhold MMT to a pregnant woman seeking treatment for opioid addiction in pregnancy, but rather, should be a consideration in her obstetrical management and in planning postnatal care for those newborns exposed. We found no differences between MMT patients and controls in the mode of delivery, with vaginal delivery being the most common. With low rates of neonatal respiratory depression at birth or need for neonatal resuscitation at birth, much of the intrapartum management for women on MMT is similar to controls.

We found that birthweight was reduced amongst newborns exposed to methadone in utero compared to neighborhood controls. This finding remained true when stratifying by other covariates between groups, namely prematurity, smoking, and advanced maternal age. There was also no statistical difference in birthweight between newborns exposed to high-versus low-dose methadone in utero. Very few newborns in either group were actually diagnosed as 'small for gestational age' (birthweight less than the $10^{\text {th }}$ percentile) at birth (5.1\% versus $\left.3.4 \%\right)$. So, while newborns exposed to methadone in utero tend to have a lower birthweights than unexposed newborns, the results may not be clinically significant, at least at birth. Future studies assessing the growth trajectory and growth potential for newborns exposed to MMT in utero, as there is very little known about catch-up growth or childhood stature following fetal methadone exposure.

Newborns exposed to MMT in utero had a significantly more admissions to the neonatal intermediate-or intensive-care units, reaching over $90 \%$. Given that there are no significant differences between groups with regards to peripartum complications, Apgar scores, or need for postnatal resuscitation, these numbers likely reflect our local hospital policy of admission of all babies at risk of NOWS to either the neonatal unit for a period of observation and possible management. It should also be noted that over half of newborns in the MMT group were admitted to NICU for under $48 \mathrm{hrs}$ of surveillance, meaning that they had low postnatal NOWS scores or showed minimal to no signs of withdrawal after birth. While NOWS was not the focus of our study, this finding would indicate that more than half of newborns, irrespective of maternal methadone dose, do not experience significant withdrawal after birth.

Given that the average cost per day of neonatal intensive/intermediate unit care in hospital in Canada is estimated $\sim 2100$ CAD per day (or $\$ 1600$ USD), the cost of care for newborns exposed to MMT in utero could be mitigated by reevaluating policies pertaining to mandatory admission to NICU for NOWS surveillance. There is excellent data about the benefits of enhanced postpartum and rooming-in care models for women on MMT and their newborns, including cost-effectiveness evaluations as well. These options should be considered as viable management solutions in order to improve maternal and neonatal outcomes, namely by reducing NOWS, and as a potential cost-savings to the healthcare system as a whole. However, given that there is no obvious correlation between methadone dosage and length of NICU stay, there remains some challenge in being able to predict which babies will exhibit significant symptoms of withdrawal compared to others and means that the postnatal care team should be vigilant with screening practices for NOWS.

There is concern that newborns exposed to high doses of methadone in utero are at increased risk of adverse neonatal outcomes, particularly postnatal respiratory depression, need of resuscitation, higher risk of NOWS, and prolonged hospital stay in neonatal intensive care. However, there remains a lack of consensus in the literature about the exact definition of high-versus low-dose MMT in pregnancy. As such, in the absence of a standardized definition, we decided to use the median dose for our study population in order to subgroup mothers and attempt to analyze the influence of methadone dosage on perinatal outcomes. Our findings are similar to what is described in the literature and suggest little relationship between daily dosage and postnatal outcomes. Specifically, we found no relationship between gestational age, incidence of preterm birth, peripartum complications or length of hospital stay in our cohort. Prolonged hospital stay of over 48 hrs at term was used as a surrogate for NOWS given that, at our hospital, newborns exposed to MMT in utero and without significant withdrawal symptoms are 'cleared' of NOWS surveillance and eligible for discharge by $48 \mathrm{hrs}$ of age. Interestingly, there was a trend towards a higher incidence of prolonged hospital stay for neonates born to mothers who received $<70 \mathrm{mg}$ of methadone per day, although our study was underpowered to find statistical significance. In fact, this tendency towards longer hospital stay amongst newborns exposed to lower doses of MMT and not the higher doses, seems opposite to what is intuitively predicted. This suggests that there are additional factors (neonatal and/or maternal), which influence the development and severity of NOWS. Furthermore, in the absence of being able to predict which newborns will develop NOWS and/or how severe, neonatal care should be planned accordingly and NOWS surveillance undertaken for all. Future studies are still required in order to identify these additional risk factors beyond maternal methadone 
dosage which influence the risk of postnatal NOWS and other complications: Once identified, any modifiable risk factors could be targeted in order to reduce frequency, severity and adverse sequelae of NOWS in hopes of improving postnatal management overall.

Strengths of this study include its matched design and attempt to account for additional socioeconomic variables that may impact neonatal outcomes in this cohort. It is also one of the few observational studies about MMT in pregnancy to focus on neonatal outcomes other than NOWS. Our case-finding process was sound and should have identified all pregnant women delivering at term who were on MMT within our center. By virtue of being a chart review, we encountered missing data/inconsistent reporting for some variables, thus limiting our analysis. There was inconsistent reporting for variables such as maternal BMI at delivery and neonatal resuscitation interventions. Another potential limitation was geographic restriction of the study population to the hospital catchment area, thereby excluding patients from rural areas because of an inability to find neighborhood controls.

While this design and strict inclusion criteria enhance reliability of our results through improving comparability of the groups, it may impact generalizability of our findings to non-urban mothers on MMT in pregnancy.

\section{Conclusion}

Newborns exposed to MMT in utero experience higher rates of preterm birth and lower mean birthweights overall, however there is no difference in perinatal complications aside from increased length of hospital stay, when compared to maternal age- and neighborhood-matched controls. Most importantly, there was no difference in survival to discharge between groups. Our study has broad implications for improving the postnatal care of this complex population, especially considering the recent rise in opioid use and increased use of MMT amongst pregnant patients. Future studies that address specific risk factors for development of NOWS and explore alternative models for postnatal care are required to insure quality care and best possible outcomes for newborns and their mothers.

\section{Acknowledgements}

The author's would like to thank Jonathan Fine and the staff of the HSC In-Patient Pharmacy for their contributions in the initial project planning and to Dr. Maggie Morris for her invaluable mentorship. We are grateful for receipt of a summer studentship salary award as financed by the Dean's Fund at the University of Manitoba Max Rady College of Medicine in order to complete portions of this work.

\section{Ethical Approval}

Ethics approval was provided by the University of Manitoba Research Ethics Board.

\section{References}

1. Soelberg CD, Brown RE Jr, Du Vivier D, et al. (2017) The US opioid crisis: current federal and state legal issues. Anesth Analg 125: 1675-1681.

2. Lind JN, Interrante JD, Ailes EC, et al. (2017) Maternal use of opioids during pregnancy and congenital malformations: A sytematic review. Pediatrics 139: e20164131.

3. Caitlin E Martin, Nyaradzo Longinaker, Terplan M (2015) Recent trends in treatment admissions for prescription opioid abuse during pregnancy. J Subst Abuse Treat 48: 37-42.

4. Services H (2014) Treatment Episode Data Set (TEDS).

5. Kaltenbach K, Berghella V, Finnegan L (1998) Opioid dependence during pregnancy. Obstet Gynecol Clin North Am 25: 139-151.

6. Patrick SW, Schumacher RE, Benneyworth BD, et al. (2012) Neonatal abstinence syndrome and associated health care expenditures. JAMA 307: 1934-1940.

7. Wong S, Alice Ordean, Meldon Kahan (2011) Substance use in pregnancy. J Obstet Gynaecol Can 33: 367-384.

8. Stanhope TJ, Gill LA, Rose C (2013) Chronic opioid use during pregnancy: Maternal and fetal implications. Clin Perinatol 40: 337-350.

9. Bell J (2014) Pharmacological maintenance treatments of opiate addiction. Br J Clin Pharmacol 77: 253-263.

10. Dole VP, Nyswander M (1966) A Medical treatment for diacetylmorphine (Heroin) addiction. J Occup Enviro Med 8: 241.

11. Jones HE, Kaltenbach K, Heil SH, et al. (2010) Neonatal abstinence syndrome after methadone or buprenorphine exposure. N Engl J Med 363: 2320-2331.

12. Jones HE, O'Grady K, Malfi D, et al. (2008) Methadone maintenance vs. methadone taper during pregnancy: maternal and neonatal outcomes. Am J Addict 17: 372-386.

13. Popova S, Rehm J, Fischer B (2006) An overview of illegal opioid use and health services utilization in Canada. Public Health 120: 320-328.

14. Wright ML, Robinson MJ (1995) Neonatal abstinence syndrome. Arch Dis Child 73: 122.

15. Mactier H, Shipton D, Dryden C, et al. (2014) Reduced fetal growth in methadone-maintaine pregnancies is not fully explained by smoking or socio-economic deprivation. Addiction 109: 482-488.

16. Cleary BJ, Donnelly JM, Strawbridge JD, et al. (2011) Methadone and perinatal outcomes: A retrospective cohort study. Am J Obstet Gynecol 204: 1-9.

17. Bell J, Towers CV, Hennessy MD, et al. (2016) Detoxification from opiate drugs during pregnancy. Am J Obste Gynecol 215: 1-6.

18. Gomez-Pomar E, Finnegan LP (2018) The epidemic of neonatal abstinence syndrome, historical references of its' origins, assessment, and management. Front Pediatr 6: 33.

19. Pryor JR, Maalouf FI, Krans EE, et al. (2017) The opioid epidemic and neonatal abstinence syndrome in the USA: A review of the continuum of care. Arch Dis Child Fetal Neonatal Ed 102: 183-187.

20. Ordean A, Kahan M, Graves L, et al. (2015) Obstetrical and neonatal outcomes of methadone maintained pregnant women: $A$ canadian multisite cohort study. J Obstet Gynaecol Canada 37: 252-257. 
21. Luty J, Nikolaou V, Bearn J (2003) Is opiate detoxification unsafe in pregnancy? J Subst Abuse Treat 24: 363-367.

22. Ordean A, Kahan M, Graves L, et al. (2013) Integrated care for pregnant women on methadone maintenance treatment: Canadian primary care cohort study. Can Fam Physician 59: E462-E469.

23. Abrahams RR, S. Ann Kelly, Sarah Payne, et al. (2007) Rooming-in compared with standard care for newborns of mothers using methadone or heroin. Can Fam Physician. 53: 1722-1730.

24. McKnight S, Coo H, Davies G, et al. (2016) Rooming-in for infants at risk of neonatal abstinence syndrome. Am J Perinatol 33: 495-501.

25. Hodgson ZG, Abrahams RR (2017) A rooming-in program to mitigate the need to treat for opiate withdrawal in the newborn. J Obstet Gynaecol Canada 34: 475-481.

26. Abrahams RR, MacKay-Dunn MH, Nevmerjitskaia V, et al. (2017) An evaluation of rooming-in among substance-exposed newborns in British Columbia. J Obstet Gynaecol Canada 32: 866871.

27. Brogly SB, Saia KA, Walley AY, et al. (2014) Prenatal buprenorphine versus methadone exposure and neonatal outcomes: $A$ systematic review and meta-analysis. Am J Epidemiol 180: 673686.

28. Cnattingius S, Forman MR, Berendes HW, et al. (1992) Delayed childbearing and risk of adverse perinatal outcome: a population-based study. JAMA 268: 886-890.

29. Moini A, Shiva M, Arabipoor A, et al. (2012) Obstetric and neonatal outcomes of twin pregnancies conceived by assisted reproductive technology compared with twin pregnancies conceived spontaneously: A prospective follow-up study. Eur J Obstet Gynecol Reprod Biol 165: 29-32.

30. Davies GA, Maxwell C, McLeod L (2010) Obesity in pregnancy. J Obstet Gynaecol Can 32: 165-173.

31. Fenton TR, Nasser R, Eliasziw M, et al. (2013) Validating the weight gain of preterm infants between the reference growth curve of the fetus and the term infant. BMC Pediatr 13: 92.

32. Duan S, Jin Z, Liu X, et al. (2017) Tobacco and alcohol use among drug users receiving methadone maintenance treatment: $A$ cross-sectional study in a rural prefecture of Yunnan Province, Southwest China. BMJ Open 7: e014643.

33. Bawor M, Brittany B. Dennis, Michael Varenbut, et al. (2015) Sex differences in substance use, health, and social functioning among opioid users receiving methadone treatment: A multicenter cohort study. Biol Sex Differ 6: 21.

34. Nahvi S, Richter K, Li X, et al. (2006) Cigarette smoking and interest in quitting in methadone maintenance patients. Addict Behav 31: 2127-2134.

35. Khanna N, Arnold S, Sadaphal S, et al. (2010) Nicotine dependence and depression among women smokers on methadone maintenance. Eur J Gen Pract 16: 222-228.

36. Wagijo MA, Sheikh A, Duijts L, et al. (2017) Reducing tobacco smoking and smoke exposure to prevent preterm birth and its complications. Paediatr Respir Rev 22: 3-10.

37. Hayashi K, Matsuda Y, Kawamichi Y, et al. (2011) Smoking during pregnancy increases risks of various obstetric complications: A case-cohort study of the Japan Perinatal Registry Network database. J Epidemiol 21: 61-66.

DOI: $10.36959 / 395 / 510$

Copyright: (c) 2020 Pylypjuk C, et al. This is an open-access article distributed under the terms of the Creative Commons Attribution License, which permits unrestricted use, distribution, and reproduction in any medium, provided the original author and source are credited. 